\title{
Misuse, perceived risk, and safety issues of household insecticides: qualitative findings from focus groups in Arequipa, Peru
}

\author{
Anika Larson ( $\sim$ larsona@uw.edu ) \\ Valerie Paz-Soldan \\ Tulane University \\ Claudia Arevalo \\ Universidad Peruana Cayetano Heredia \\ Joanna Brown \\ Universidad Peruana Cayetano Heredia \\ Carlos Condori-Pino \\ Universidad Peruana Cayetano Heredia \\ Michael Levy \\ University of Pennsylvania \\ Ricardo Castillo Neyra \\ University of Pennsylvania
}

University of Washington https://orcid.org/0000-0001-9499-8278

\section{Research}

Keywords: Insecticides, focus groups, poisoning, qualitative research,vector control

Posted Date: May 5th, 2020

DOl: https://doi.org/10.21203/rs.3.rs-25073/v1

License: (c) (i) This work is licensed under a Creative Commons Attribution 4.0 International License. Read Full License 


\section{Abstract}

Background The current body of research on insecticide use in Peru deals primarily with application of insecticides offered through Ministry of Health-led campaigns against vector-borne disease. However, there is a gap in the literature regarding the individual use, choice and perceptions of insecticides which may influence uptake of public health-based insecticide initiatives and contribute to the thousands of deaths annually from acute pesticide poisoning in Peru.

Methods Residents $(n=49)$ of the Alto Selva Alegre and CC districts of peri-urban Arequipa participated in seven focus group discussions (FGD). Using a FGD guide, two facilitators led the discussion and conducted an activity in which participants advertised insecticides (physically available during the FGD) to elicit perceptions of currently available insecticides. Focus group content was transcribed, and qualitative data were analyzed with Atlas.ti and coded using an inductive process to generate major themes related to use and choice of insecticides, and perceived risks associated with insecticide use.

Results There was not consensus on a preferred form, strength, or brand of insecticides; valued characteristics such as strength and effectiveness were often associated with negative characteristics such as odor and health impacts. The perceived risks associated with insecticides included both shortand long-term health impacts, and safety for children emerged as a priority. However, some participants reported applying insecticides directly to children and their beds. Some participants attempted to reduce the risk of insecticide use with informal, and likely ineffective, personal protective equipment and by timing application when household members were away. "Agropecuarios" (agricultural supply stores) were considered a trusted source of information about insecticides and their health risks.

Conclusions It is crucial to characterize misuse and perceptions of health impacts and risks of insecticides at the local level, as well as to find common themes and patterns across populations to inform national and regional programs to prevent acute insecticide poisoning and increase community participation in insecticide-based vector control campaigns. We detected risky practices and beliefs about personal protective equipment, risk indicators, and safety levels that could inform such preventive campaigns, as well as trusted information sources such as agricultural stores for partnerships in disseminating information.

\section{Introduction}

Studies of household insecticide use and community fumigation in Peru have historically focused on the chemical control of vector-borne disease targeting mosquitoes and triatomines (1-7). Household insecticides can present a serious health risk for household members, particularly children $(8,9)$. It is important to characterize holistic household insecticide use and perceptions of insecticide safety and toxicity in Peru to incorporate safe insecticide practices into vector control campaigns.

Acute pesticide poisoning is a widespread and underreported problem in Latin America (8). The Peruvian Ministry of Health documented a total of 2,489 deaths from acute pesticide poisoning in 2017 alone; 
$7.9 \%$ were classified as accidental and unrelated to occupational exposure to pesticides, and $8.5 \%$ occurred in homemakers and 3.2\% infants (9). Acute pesticide poisoning of children can be caused by household behaviors including storage of insecticides in unmarked containers and using insecticides directly on children (8).

Household insecticide use and uptake of insecticide campaigns in Peru has been studied predominantly in response to serious health threats from vector-borne disease $(1-3,5,6,10)$. In Arequipa and surrounding areas where the current study was conducted, insecticide research efforts have focused particularly on addressing triatomine, or "kissing bug," populations in response to Chagas disease $(1,11-$ 14). One residential insecticide spray campaign in peri-urban Arequipa in 2012 reached only $66 \%$ of homes, and a study found that primary reported barriers included practical concerns such as spray times coinciding with work obligations and difficulties preparing the home, as well as concerns about the health impacts of insecticides and a perception that the campaign was not necessary (1). Though unreported in that publication, one of the less reported comments from household members refusing spraying was that they had used some form of vector control product on their own, which led to our concern about types and amounts of insecticide that might be in use in this region. In Iquitos, Peru, in addition to participating in fumigation campaigns for mosquito control, participants reported sprinkling or rubbing petroleum and/or creoline almost daily on their floors and door/window frames, as well as other cleaning products and insecticide sprays indoors to combat mosquitoes (15).

Our study objective is to examine how household members in peri-urban Arequipa, Peru choose and use different insecticides in the home and explore what guides their decision-making process as they choose insecticides, using focus group discussions. A greater understanding of perceptions and use of insecticides on the household level will help public health officials and policymakers to provide helpful and relevant tools and education to promote safe and effective insecticide use and combat vector-borne disease in vulnerable communities.

\section{Methods}

\section{Study setting}

The study was conducted in Alto Selva Alegre (ASA) (population for 2017: 85,870; density: 12,302 people/km2) and Cerro Colorado (CC) (population for 2017: 197,954; density: 1,131 people/km2), two of the 14 districts of the city of Arequipa. Arequipa, Peru's second largest city, is home to 969,000 people and is situated at $\sim 2,300$ meters above sea level. The 14 districts in Arequipa vary in human population size, house density, and socioeconomic status. They are formed by contiguous neighborhoods and this variation is usually associated with the level of urbanization of those neighborhoods, with lower levels or urbanization in peri-urban (peripheral) areas. ASA and Cerro Colorado span the gradient of urbanization. In our study, participants represented peri-urban residential areas of the city of Arequipa.

\section{Sampling strategy}


Purposive sampling was used to select participants for seven focus group discussions (FGD). We invited all those who lived in ASA or CC and who had reported bed bug infestations in the past two years (approximately 15 individuals). We also recruited households with other types of insect infestations during the last 2 years (e.g. flies, cockroaches) by knocking on doors in houses 4 blocks around the bed bug infested house, but not within the same block. All participants were residents of the districts of ASA or CC. Recruitment criteria included having had a domestic insect infestation - whether bed bug or other - during the last two years, being over 18 years of age and willing to consent to the study.

\section{Recruitment approach}

The recruitment strategies described in the previous section were applied until the quota (8 to 12 participants per focus group) was reached. In case people were unable to attend or chose not to participate in the focus groups, the team invited more than 12 individuals to ensure a minimum number of participants. An additional focus group was carried out due to low attendance in one group.

Participants were explained the study goals by the recruitment team and who obtained written informed consent.

Participants were informed about the date and time of the FGD and confirmed their assistance. Arrangements were made to pick them up from their houses and take them to the FGD locations. Participants were given compensation for transportation back home.

\section{Study population}

Forty-nine individuals, between 18 and 81 years of age, participated in seven FGDs (4 groups in ASA and 3 groups in CC) over four days. Seventy three percent of participants were female $(n=36)$.

\section{Study Design}

A FGD guide was used to facilitate the data collection. The themes covered in the FGD guide included experiences with insect infestations in homes, information about use of insecticides and other pest control methods, decision-making and practices associated to the purchase and use of insecticide products, and risks perceptions about these products.

Prior to the FGD, the research team purchased different types of insecticides available in stores, in local markets, as well as sold by "agropecuarias" (small or medium size agricultural supply stores that sell manure, chemical fertilizer, seeds, as well as pesticides, and are usually located in the periphery of the city). During the FGD, participants were asked to break into groups of two, pick from the various insecticides set up on a table, and they were given time to prepare a short marketing presentation about the product they chose, based on what they knew or could read about on the product. The research team split to be able to listen to the conversations of the various pairs. Then all FGD participants were reconvened and they were asked to "sell" their product to the rest, while the research team noted what insecticide product elements were highlighted during these short presentations. 
The FGDs were facilitated by a social scientist and a research assistant, with technical support of an infectious disease epidemiologist and a biologist with years of experience in insect vector control. There were two notetakers who are also part of the research team. All research team members are Peruvian, and half of the team resides in Arequipa.

\section{Data management and analysis}

With written informed consent, all FGD were digitally audio-recorded and transcribed; detailed notes were also taken throughout. An inductive coding process was used: we first grounded ourselves in the data to explore the topics that would emerge, not knowing what to expect (Bernard, 2006). Although the main themes being explored were established prior to the FGD, all subcodes were then developed based on the emerging themes. Focus group transcripts were imported into ATLAS.ti [48] and coded into the four major themes: problems with insects in the home (which will be published separately); choice of insecticides; use of insecticides; risks of the use of insecticides. As described, the subcodes were developed based on what emerged by theme. Main findings of interest were summarized using the coded material as well as the original transcripts. Relevant quotes are also presented by theme.

\section{Results}

Main themes that emerged in the focus group discussions are reported by sections. Key findings included safety/health, effectiveness, convenience and low odor as important factors in choosing insecticides, and "agropecuarios" (agricultural supply stores) as trusted sources of information. There was widespread misuse of insecticides in the home, including informal fumigation, combining insecticides or mixing with other materials, direct use on children and animals, and inadequate use of safety equipment; participants reported symptoms consistent with acute pesticide toxicity when using insecticides as well as human and animal fatalities related to insecticide ingestion.

\subsection{Proper and Improper Insecticide Use 3.1.1. Where Insecticides are Used}

Insecticides were used in numerous areas of the home including bedrooms, kitchens, bathrooms, and yards/patios. Powder insecticides, such as the popular "Chica Verano" (deltamethrin 5\% soluble powder), were applied as indicated (dissolved in water) or pure as a powder (not the use indicated on the instructions) (16). Spray insecticides were used as needed or for regular informal fumigation, ranging from every 2 weeks to every 2 months. A typical scenario was to spray within in the home, leave the homes closed for a period of time while children family members were away, and ventilate on return. Several participants also reported fumigation from the government spray campaigns against "chirimachas" (triatomines).

There was also a reports of insecticide spraying used directly on dining plates: 
While working as a cook, we used it [insecticides] even on dishes... The lady told me "bring the Raid, quickly" and we didn't even close the house. We sprayed it on the plates and then sat and talked... I asked her, "are we going to rinse the plates?" but she said that by the time we serve food it will have evaporated."

\subsubsection{Frequency of Insecticides Use}

Participants would wait from half an hour to the following day before repeating insecticide application for an ongoing infestation. Some participants reported that they would switch insecticides if the first did not work, combine the initial insecticide with another, use multiple targeted insecticides for multiple insect species, or mix insecticide with household cleaners. Others avoided combining insecticides due to concerns about health, decreased efficacy, or "resistance".

Facilitator: Have you ever used Chica Verano mixed with spray?

Woman 1: Spray with Poett [brand of household cleaner].

Facilitator: Spray with Poett. But have you ever used it, has anyone used insecticide combined with another?

Woman 2: With "Folidol" [likely Methyl Parathion 20\%].

-Cerro Colorado

\subsubsection{How Insecticides are Used}

Several cases of insecticide application directly to children and animals were reported. One participant used powdered deltamethrin $5 \%$ directly in her daughter's hair against lice, tying a plastic bag over her daughter's eyes as protection.

Woman: My little daughter [ 3 years old, but this was done when she was 2.5 years old approximately], with this ["Chica Verano" (deltamethrin 5\%)] I have powdered her all, they have made me shampoo of this; 'for the hair' they said, 'for fleas', I put it on her ... everything has disappeared, even the nits.

Facilitator: Did you do it with dry powder or water?

Woman: From here, I tied [her hair] like this and I put it as powder, if not it will get in her eyes and mouth.

-Cerro Colorado

Facilitator: Has anyone else used anything else apart from Nopucid [lice shampoo]?

Woman: My sister lives in Satipo [another city] and there are a lot [of lice] and I have seen her use an iron comb, I have also seen how she has reached despair and has put a powder for insects on the baby's head. 
A few participants who sold or worked with bulk food products reported placing a vaporizing pill (a phosphine pellet or tablet designed to release insecticidal vapors for warehouse use) under products to keep insects away. For protection, participants who reported using phosphine used a piece of toilet paper to protect their skin and did not mention any gas monitoring. In one case, the participant (Man 1, CC) reported using it in a warehouse overnight. Another participant (CC) left the vaporizing pill in a store during working hours; when asked if people in the same room with phosphine could be affected, he explained that you could not touch it but may have been unaware of the inhalation risk (or chose to ignore it).

Man 1: I wrap it in a piece of toilet paper and put it between the sacks; you put it under a few of them and the smell penetrates, everything goes away.

$-\mathrm{CC}$

Participants from both districts reported that they had or would use insecticides in beds, including children's beds, but responses varied and participants from two focus groups agreed they would not immediately apply insecticide directly to a mattress, even if children were showing bug bites.

"I feel the fleas and run to buy and spray it. My children are sleeping and [I spray it] not too close to the face but near the feet..."

- Woman, ASA

Cleaning a bed for bedbugs was described in both regions and across multiple focus groups as a multistep process that could involving taking the bedding and mattress out to the sun, washing bedding with bleach, using insecticide on the wooden bedframe, and spraying alcohol in the bed. As a last resort, participants also reported burning bedding and mattresses. One participant reported burning her own mattress in desperation to rid her bed of bedbugs after fumigating with "Baygon" every 2-3 days for three months.

Woman: The mattress, ah no, I burned the mattress.

Facilitator: You have burned it?

Woman: Yes, I have burned it, the bed, the mattress, everything because I was desperate. I burned everything.

-ASA

Other alternative methods of controlling insects including cleaning and disposing of trash, using noninsecticide chemicals such as alcohol, bleach, detergent, gasoline and soap, physical barriers or tools such as screens, flyswatters, water bags (placed on or hung over tables to "scare" flies away), and 
mosquito repellant. Herbal and natural methods of repelling insects included using herbs such as eucalyptus, mole, and muña [similar to mint]; smoke, particularly of those herbs; ash, talc, and hot water.

Well, in the house, since I'm an old lady, I burn eucalyptus.

-Woman, CC

\subsection{Perceived Risks of Insecticide Use 3.2.1. Health Risks of Insecticides}

Participants reported that the short-term risks of insecticide exposure or ingestion included headaches, trouble breathing, pain in the hands, burning eyes, irritation, dizziness, vomiting, and even death of animals or people. Allergic reactions were a common concern, especially for children. Moreover, participants recognized a difference in individuals' responses to insecticides - that the response to "Chica Verano" (deltamethrin 5\%) might be fine for some, but that others might be sensitive to it.

Alarmingly, participants in both regions reported personal experiences with symptoms consistent with acute pesticide toxicity, including headache, vision changes and difficulty breathing.

Man 1: Because if you stay a bit [in a room with insecticide], it harms you...

Woman: And your head hurts.

Man 2: Your head hurts, and your vision [eyes] too.

$-\mathrm{CC}$

Woman 1: When I use it, with that odor, my head hurts, it hurts my hand.

Facilitator: It gives you a headache. Any worry about using the insecticide?

Woman 2: Because of the vision.

Facilitator: What happens with the vision?

Woman 2: It burns.

-ASA

Several participants reported fatal incidents related to insecticide use. One participant described a friend who drank Malathion (organophosphate insecticide), thinking it was a medicine, and died. Another participant reported that after using a particular insecticide, her daughter became dizzy and her dog vomited; another reported that her puppy died from ingesting "Chica Verano". 
"I have had [bad experiences], with Chica Verano; trying to kill flies, I killed my puppy... And I took it to the veterinarian and even with a vet, I couldn't save my puppy."

-Woman, CC

Children were considered inherently more vulnerable to the health risks of insecticides. Contributing factors included their behavior, such as picking up or swallowing insecticides, as well as the possibility of allergies and reactions to insecticides that they had never been exposed to before.

"I have had to fumigate in my work, but we were adults. But with children, we don't know if they have allergies, because when they're born you see the child but you don't know what sickness they will have or what allergies they're going to present, or what products will harm the child, so there's no guarantee, you have to take them outside."

-Woman, ASA

One participant asked what toxicity meant and whether the odor and strength of an insecticide was a proxy of the toxicity. Several participants reported that all insecticides inherently presented a risk for health, because they were designed to be lethal to living things.

Man: A question, for my knowledge, what is meant by toxic? The lady says it's toxic, what should I understand?

Facilitator: I don't know, what do you understand it to mean?

Man: I don't know if I have it right

Facilitator: Yes, yes, go on.

Man: My dad used the baygon spray [insecticide spray made by "Baygon"], so he would protect himself, but it left a horrible odor, an odor that I couldn't be around, so I couldn't be there - it was a toxic atmosphere for me because it didn't allow me to breathe for example... When I mix this product, it doesn't have an odor, so from my point of view, is it less toxic or not toxic because I can be in the environment without using any type of protection?

Facilitator: And how do you know you don't need to use protection?

Man: Because it doesn't say here either, "I let myself be guided by the smell," by the strength.

$-\mathrm{CC}$

\subsubsection{Risk Prevention during Insecticide Use}

To reduce the risks associated with insecticides, some participants described using informal or homemade personal protective equipment; keeping insecticides stored in the home out of reach of 
children; timing their application of insecticides when family members were out of the house; and using insecticides as directed and seeking information on which insecticide to choose. However, risk prevention reporting was likely to be biased towards those that take precautions, and precautions described were frequently inadequate (e.g. the use of bags or handwashing for hand protection) and did not fully protect participants from acute exposure to insecticides.

Reported informal personal protective equipment included use of gloves or bags tied over one's hands and glasses, and masks or rags over the face. Another participant explained that he did not use gloves for one insecticide because it was a powder, and his decision regarding need for insecticide protection was determined by the strength of the odor: if he could stand to be in the environment with the insecticide he did not need protection. One reported that she thought the majority of people did not use these precautions.

Woman: I put bags [on my hands]. If I don't have any, I just wash my hands - water with soap - and I put on my glasses.

\section{Facilitator: Glasses?}

Woman: Because I have bad eyesight, a mask.

Facilitator: You use a mask?

Woman: When you don't have money, what are you going to do?

$-\mathrm{CC}$

To prevent harm to children, some participants described keeping insecticides in high places or in hidden areas of the house, as well as spraying when children were out of the house. However, one participant said that her daughter would touch the insecticides regardless of precautions. Another woman described her daughter touching walls wet with insecticides after a government-led fumigation campaign.

"You have to prepare chica verano, it takes a little more time, of course it's good too! Because if there are small children, we are freed from them being intoxicated... I have a little girl that is very naughty. In order to sprinkle this, I have her by my side and I go with her. But all the same she touches because she is very curious. Children don't warn you the moment they're going to do something, in one second, boom [they are up to something]."

-Woman, ASA

Some participants reported knowing how to use insecticides safely by following the directions on an insecticide and reading warning labels. Others said that before using a product, they would seek out information, usually from trusted sources such as agricultural stores. However, during the focus groups facilitators noted that few participants could find the warning instructions in the actual bottles, cans and bags of commercial insecticide provided. 


\subsection{Choosing Insecticides}

\subsubsection{Ease of Use}

Spray and powder insecticides were the two major forms of insecticides discussed. Spray insecticides were considered quicker and more convenient, since they did not require preparation and could be used immediately on seeing an insect, while powder insecticides had to be mixed and prepared in order to convert them into a spray.

Facilitator: But do you use the one that is a spray or sometimes you use another one.

Man: No! Just the spray.

Facilitator: And why do you always use the spray?

Man: For me it is more comfortable and more practical.

$-\mathrm{CC}$

\subsubsection{Safety}

Three themes emerged associated to insecticide safety: stronger insecticides were considered more harmful to health; the ease of keeping the insecticide away from children (including a safety lock and whether the packaging was colorful and enticing); and permanence in the environment after use. Powdered insecticides were referred to as a safety risk because they remain in the home and can be found by children in contrast to spray that evaporates. However, "Chica Verano," a powder insecticide used by participants in both regions, was referred to as non-toxic by multiple participants during the advertising group activity and one participant used it directly on her daughter as discussed in Sect. 3.1.3.

Facilitator: [In the activity] we saw that many people preferred the sprays over the bags of powder. Why would they choose the sprays more than the powders?

Man: For the practicality of the sale.

Woman: Practical. When we have children, we don't use the powder, they can grab it. In contrast, with the spray they go to school and we take advantage and spray it.

$-\mathrm{CC}$

\subsubsection{Strength}

The strength or effectiveness of an insecticide was an important factor and was associated by many respondents to the odor: the stronger the odor, the stronger the insecticide and the more harmful to people and long-lasting it would be. In general, "Baygon", "Raid", and "Sapolio" were perceived as the most potent and strongest brands. Some participants purchased insecticides to the insects they were designed to kill, 
especially for spiders, mosquitoes, flies, and roaches, but certain insecticides were considered "broadspectrum" and it was agreed that if an insecticide could kill cockroaches, it could kill any bug.

\subsubsection{Cost}

Lower priced insecticides were generally associated with lower quality, including the possibility that insecticides were expired or not genuine products or were more harmful. One participant noted both that all cheap products were harmful, but also that high prices or brand names were not necessarily an indication that a product worked.

"Maybe because it's more economical, so of course I say 'No!' If it's cheap, it is not so effective, I save a little more and I buy a better one."

-Man, CC

\subsubsection{Sources of Insecticides and Information}

The most trusted sources of information and insecticides were "agropecuarias," or agricultural feed stores. Agropecuarias gave advice and recommendations regarding the most appropriate insecticide, as well as advising on possible health impacts. Street vendors were considered cheap sources of insecticides but possibly unreliable; participants reported that the insecticides could be "bamba" (knockoffs) or expired.

Facilitator: Have you gone to the street sellers or agriculture stores? Have you asked: what do you recommend to me, what can I use, what is best, what is most dangerous?

Woman 1: We guide ourselves by the labels that say 'for flies, cockroaches'. If we want for rats, we go and look, but like they say, it is better to go to an agriculturist and they will give us better information.

Woman 2: I once went and they asked me if anyone had allergies in the house. Do you have young children they asked? Because they say you can't use it if anyone in your family gets welts. I told them no.

-ASA

Woman: We go to the market, we see that there are a ton of insecticide vendors. Those little packets, sometimes there are knockoffs, they cost less. It costs $\mathrm{S} / .1 .00, \mathrm{~S} / .1 .50$ [US\$0.30-0.50). In order to try it out, I buy it, but they are not effective."

-Woman, ASA

\section{Discussion}

The findings of this study raise significant concerns for insecticide safety in peri-urban Arequipa, including the availability of off-label pesticides and misuse within homes and workplaces. Home storage of pesticides, exposure to indoor pesticides, and direct contact with pesticides (including direct use of 
deltamethrin $5 \%$ on children) were all identified in this study. These are known risk factors for various health impacts among children including acute pesticide poisoning, childhood cancers and developmental toxicity $(8,17,18)$; a 5-year-old in Peru died in 2010 after drinking an unmarked bottle of "Chica Verano" (deltamethrin 5\%) (19). Participants reported symptoms consistent with acute pesticide poisoning (headache, vision changes, and difficulty breathing)(20) when using insecticides as well as human and animal fatalities related to insecticide ingestion.

The widespread availability and incorrect use of pesticides for use in households and small businesses is concerning, especially in the context of family-owned businesses where children may be exposed to pesticides used to protect agricultural products. Safety measures were generally informal and included makeshift hand and face coverings in place of personal protective equipment. A pellet form of phosphine insecticide was used by participants with only paper as protective equipment to fumigate stored food products, with a participant commenting incorrectly that you could be in the same room with it as long as you did not touch it.

One opportunity for change may be the promotion of purchasing insecticides through reliable sources. The National Inventory of Pesticides in Peru has identified the presence and sale of expired, contraband, and false or adulterated pesticides (21). The practice of purchasing insecticides from "ambulatory" vendors, which were considered cheap, may expose household members to subpar or mislabeled insecticides as well as bypassing health and safety regulation of the products. In contrast, participants reported that "agropecuarias" or agricultural supply stores are a source of information about health and use of insecticides as well as a vendor. With additional training to the workers, these stores may be valuable partners to disseminate information in future campaigns.

The ultimate goal for reduction of pesticide poisoning from a global health perspective should be the phasing out of the WHO Class I and II pesticides (deltamethrin, for example) supported by the introduction of safe alternatives $(22,23)$. The health benefits of insecticide use against disease vectors such as mosquitoes and triatomines should be weighed relative to the possibility of misuse and resistance $(24,25)$. These risk-benefit analyses are further complicated by insects such as bed bugs, which may not present quantifiable health risks but represented a major nuisance and psychological burden to community members in our study (26).

Strategies such as integrated vector control can and should be used to maximize the long-term benefits of insecticides against vector-borne disease while minimizing risk $(27,28)$. If possible, community-based vector control campaigns should take into account current household insecticides use when planning campaigns. Of the major household brands identified in the current study many include pyrethroid insecticides, a class that is widely used in the Americas for control of Chagas disease (25) and presents a risk for development of resistance as well as acute health impacts $(25,29,30)$. Future campaigns should build awareness of safe use of household insecticides and prioritize community values such as safety, convenience, health and low odor of insecticides for household members to develop campaigns that can be safely incorporated into current household practices. 


\section{Conclusions}

Incorrect use of insecticides is common in households and small businesses in Arequipa, Peru, subjecting those exposed to toxic levels of these products based on their descriptions of experiences when using these. Vector control campaigns utilizing insecticides in this region should recognize that household insecticide use may already include informal fumigation and, in some cases, direct application to animals and children without adequate protection. Campaigns should also be cognizant of concerns about both immediate safety and health impacts of insecticide use and should work with trusted sources, such as "agropecuarias," to disseminate information on insecticides and their safe and correct use.

\section{Abbreviations}

FGD

Focus Group Discussion

\section{Declarations}

\section{Ethics approval and consent to participate}

Institutional Review Board approval was obtained from Universidad Peruana Cayetano Heredia (approval identification number: 65369), Tulane University (approval identification number: 14-606720), and University of Pennsylvania (approval identification number: 823736). Written consent to participate and to be audiotaped was obtained from all focus group participants.

\section{Consent for publication}

All authors consent to publication and consent forms will be submitted to BMC Environmental Health.

\section{Availability of data and materials}

Deidentified focus group transcripts have been made available as an attached file.

\section{Competing interests}

The authors declare no competing interests.

\section{Funding}

This study was supported by Eunice Kennedy Shriver National Institute of Child Health and Human Development (R01HD075869) and the National Institute of Allergy and Infectious Diseases 
(R01Al146129) for VPS, MZL, and RCN, as well as the Fogarty International Center/NIH K01Al139284 for RCN.

\section{Authors' contributions}

Study design was conducted by VPS, MZL and RCN. Focus groups were conducted by VPS, JB, CC, and RCN. Qualitative analysis was performed by JB, CA, AL, and VPS. The Background, Results, Discussion and Conclusions sections were drafted by AL with contributions from VPS and RCN. The Methods section was drafted by VPS with contributions from RCN and AL. All authors reviewed, edited, and approved the final draft of the manuscript.

\section{Acknowledgements}

The authors would like to acknowledge and extend thanks to the Gerencia Regional de Salud de Arequipa (GRSA).

\section{Authors' information}

Anika Larson, Email: larsona@uw.edu

Valerie Paz-Soldan, Email: vpazsold@tulane.edu

Claudia Arevalo,Email: arenica2@gmail.com

Joanna Brown, Email: joannabrown84@gmail.com

Carlos Condori-Pino, Email: carlosedcopino@gmail.com

Michael Z. Levy: mzlevy@pennmedicine.upenn.edu

Ricardo Castillo-Neyra, Email: cricardo@upenn.edu

\section{References}

1. Paz-Soldán VA, Bauer KM, Hunter GC, Castillo-Neyra R, Arriola VD, Rivera-Lanas D, et al. To spray or not to spray? Understanding participation in an indoor residual spray campaign in Arequipa, Peru. Glob Public Health. 2018 Jan;13(1):65-82.

2. Paz-Soldan VA, Bauer KM, Lenhart A, Cordova Lopez JJ, Elder JP, Scott TW, et al. Experiences with insecticide-treated curtains: a qualitative study in Iquitos, Peru. BMC Public Health [Internet]. 2016 Jul 16 [cited 2018 Jun 20];16. Available from:

https://www.ncbi.nlm.nih.gov/pmc/articles/PMC4947330/.

Page 15/18 
3. Paz-Soldan VA, Bauer K, Morrison AC, Cordova Lopez JJ, Izumi K, Scott TW, et al. Factors Associated with Correct and Consistent Insecticide Treated Curtain Use in lquitos, Peru. PLoS Negl Trop Dis [Internet]. 2016 Mar 11 [cited 2018 Jun 20];10(3). Available from:

https://www.ncbi.nlm.nih.gov/pmc/articles/PMC4788147/.

4. Stoddard ST, Wearing HJ Jr, Morrison RCR, Astete AC, Vilcarromero H. S, et al. Long-Term and Seasonal Dynamics of Dengue in Iquitos, Peru. PLOS Neglected Tropical Diseases. 2014 Jul;17(7):e3003. 8(.

5. Grietens KP, Muela Ribera J, Soto V, Tenorio A, Hoibak S, Aguirre AR, et al. Traditional Nets Interfere with the Uptake of Long-Lasting Insecticidal Nets in the Peruvian Amazon: The Relevance of Net Preference for Achieving High Coverage and Use. PLoS One [Internet]. 2013 Jan 2 [cited 2018 Jun 13];8(1). Available from: https://www.ncbi.nlm.nih.gov/pmc/articles/PMC3534704/.

6. Iyer M, Skelton J, de Wildt G, Meza G. A qualitative study on the use of long-lasting insecticidal nets (LLINs) for the prevention of malaria in the Peruvian Amazon. Malar J [Internet]. 2019 Sep 2 [cited 2020 Jan 4];18. Available from: https://www.ncbi.nlm.nih.gov/pmc/articles/PMC6721337/.

7. Newell I, Wiskin C, Anthoney J, Meza G, de Wildt G. Preventing malaria in the Peruvian Amazon: a qualitative study in Iquitos, Peru. Malar J [Internet]. 2018 Jan 16 [cited 2019 Jul 23];17. Available from: https://www.ncbi.nlm.nih.gov/pmc/articles/PMC5771103/.

8. Laborde A, Tomasina F, Bianchi F, Bruné M-N, Buka I, Comba P, et al. Children's Health in Latin America: The Influence of Environmental Exposures. Environ Health Perspect. 2015 Mar;123(3):2019.

9. Nayhua Guerrera L. Vigilancia epidemiológica del riesgo de exposición e intoxicación por plaguicidas Perú, 2018. [Internet]. Centro Nacional de Epidemiología, Prevención y Control de Enfermedades, MINSA. Available from: http://www.dge.gob.pe/portal/docs/tools/Vplaguicidas040718.pdf.

10. Buttenheim AM, Paz-Soldán VA, Castillo-Neyra R, Toledo Vizcarra AM, Borrini-Mayori K, McGuire M, et al. Increasing participation in a vector control campaign: a cluster randomised controlled evaluation of behavioural economic interventions in Peru. BMJ Glob Health. 2018;3(5):e000757.

11. Levy MZ, Bowman NM, Kawai V, Waller LA, Cornejo del Carpio JG, Benzaquen EC, et al. Periurban Trypanosoma cruzi-infected Triatoma infestans, Arequipa, Peru. Emerg Infect Dis. 2006 Sep;12(9):1345-52.

12. Barbu CM, Buttenheim AM, Pumahuanca M-LH, Calderón JEQ, Salazar R, Carrión M, et al. Residual Infestation and Recolonization during Urban Triatoma infestans Bug Control Campaign, Peru1. Emerg Infect Dis. 2014 Dec;20(12):2055-63.

13. Levy MZ, Barbu CM, Castillo-Neyra R, Quispe-Machaca VR, Ancca-Juarez J, Escalante-Mejia P, et al. Urbanization, land tenure security and vector-borne Chagas disease. Proc Biol Sci. 2014 Aug 22;281(1789):20141003.

14. Delgado S, Castillo Neyra R, Quispe Machaca VR, Ancca Juárez J, Chou Chu L, Verastegui MR, et al. A History of Chagas Disease Transmission, Control, and Re-Emergence in Peri-Rural La Joya, Peru. 
PLoS Negl Trop Dis [Internet]. 2011 Feb 22 [cited 2018 Jun 13];5(2). Available from: https://www.ncbi.nlm.nih.gov/pmc/articles/PMC3042997/.

15. Paz-Soldan VA, Plasai V, Morrison AC, Rios-Lopez EJ, Guedez-Gonzales S, Grieco JP, et al. Initial Assessment of the Acceptability of a Push-Pull Aedes aegypti Control Strategy in Iquitos, Peru and Kanchanaburi, Thailand. Am J Trop Med Hyg. 2011 Feb;4(2):208-17. 84(.

16. CHICA VERANO POLVO SOLUBLE X 12 GR. [Internet]. [cited 2020 Apr 2]. Available from: https://eochperu.com/index.php?route=product/product\&product_id=335.

17. Benitez-Leite S, Macchi M, Acosta M. Congenital Malformations Associated with Toxic Agricultural Chemicals. Pediatria [Internet]. 2007 [cited 2019 Mar 19];34(2). Available from: http://scielo.iics.una.py/scielo.php?script=sci_arttext\&pid=S1683-98032007000200002.

18. Chen M, Chang C-H, Tao L, Lu C. Residential Exposure to Pesticide During Childhood and Childhood Cancers: A Meta-Analysis. Pediatrics. 2015 Oct 1;136(4):719-29.

19. Piura. Menor muere al ingerir insecticida por error [Internet]. Diario Correo. 2017 [cited 2019 Aug 4]. Available from: https://diariocorreo.pe/edicion/piura/piura-menor-muere-al-ingerir-insecticida-porerror-733686/.

20. PSEP.: Tutorials/Slide sets :: Pesticide Applicator Core Tutorial :: Module 9: Symptoms of Pesticide Poisoning [Internet]. [cited 2020 Apr 2]. Available from: http://psep.cce.cornell.edu/Tutorials/coretutorial/module09/index.aspx.

21. Inventario Nacional de Plaguicidas COP. 2006. [Internet]. SINIA I Sistema Nacional de Información Ambiental. [cited 2019 Aug 5]. Available from: https://sinia.minam.gob.pe/documentos/inventarionacional-plaguicidas-cop-2006.

22. Konradsen F, van der Hoek W, Cole DC, Hutchinson G, Daisley H, Singh S, et al. Reducing acute poisoning in developing countries-options for restricting the availability of pesticides. Toxicology. 2003 Nov 5;192(2):249-61.

23. WHO | The WHO Recommended Classification of Pesticides by Hazard [Internet]. WHO. [cited 2019 Oct 10]. Available from: http://www.who.int/ipcs/publications/pesticides_hazard/en/.

24. Bouwman $\mathrm{H}$, Kylin H, (Riana) Bornman M. The paradox of the effectiveness of IRS insecticides (including DDT) and its impacts on human health - what can we fix if it isn't broken? Malar J. 2012 Oct 15;11(1):P14.

25. Berg H, van den, Zaim M, Yadav RS, Soares A, Ameneshewa B, Mnzava A, et al. Global Trends in the Use of Insecticides to Control Vector-Borne Diseases. Environ Health Perspect. 2012 Apr;120(4):577.

26. Berg R. INSIDE THE PROFESSION: Bed Bugs: The Pesticide Dilemma. J Environ Health. 2010;72(10):32-5.

27. Tillett T. Optimizing Insecticide Resources: Global Trends in Vector Control. Environ Health Perspect. 2012 Apr;120(4):a164.

28. Golding N, Wilson AL, Moyes CL, Cano J, Pigott DM, Velayudhan R, et al. Integrating vector control across diseases. BMC Med [Internet]. 2015 Oct 1 [cited 2019 Mar 19];13. Available from: https://www.ncbi.nlm.nih.gov/pmc/articles/PMC4590270/. 
29. Saillenfait A-M, Ndiaye D, Sabaté J-P, Pyrethroids: Exposure and health effects - An update. International Journal of Hygiene and Environmental Health. 2015 May 1;218(3):281-92.

30. ATSDR - Public Health Statement. Pyrethrins and Pyrethroids [Internet]. [cited 2019 Mar 20]. Available from: https://www.atsdr.cdc.gov/phs/phs.asp?id=785\&tid=153. 